\title{
Scalable Integration of Coplanar Heterojunction Monolithic Devices on Two-Dimensional $\mathrm{In}_{2} \mathrm{Se}_{3}$
}

\author{
Subhrajit Mukherjee ${ }^{\dagger}$, Debopriya Dutta ${ }^{\dagger}$, Pranab K. Mohapatra ${ }^{\ddagger}$, Lital Dezanashvili $^{\ddagger}$, Ariel Ismach $^{\ddagger}$ \\ and Elad Koren ${ }^{\dagger, *}$ \\ ${ }^{\dagger}$ Nanoscale Electronic Materials and Devices Laboratory, Faculty of Materials Science and Engineering, \\ Technion - Israel Institute of Technology, Haifa, 3200003, Israel. \\ ${ }^{\ddagger}$ Department of Materials Science and Engineering, Tel Aviv University, Ramat Aviv, Tel Aviv, 6997801, Israel.
}

Table of content:
A. Growth setup and morphology of $\operatorname{In}_{2} S e 3:$ Figure $S 1 \&$ S2
B. Effect of laser dose on $\mathbf{I n}_{2} \mathrm{Se}_{3}$ : Figure S3 \& S4
C. Laser induced simulated temperature rise on $\operatorname{In}_{2} \mathrm{Se}$ : Figure S5
D. Effect of $\boldsymbol{e x}$-situ anneling: Figure S6
E. Effect of gaussian laser beam prolife on materials conversion: Figure S7
F. Raman maps for low intensity laser illumination: Figure S8
G. Laser exposure intensity calibration by KPFM: Figure S9
H. Comparison of laser exposure effect on sample and substrate: Figure S10
I. Surface potential, electric field and conductivity of FETs: Figure S11, S12 \& S13
J. Photoresponse characteristics of the FETs: Figure S14, S15 \& S16
K. 2D layer transfer illustration: Figure S17
L. Raman characteristics of $\mathrm{In}_{2} \mathrm{Se}_{3}$ before and after wet-transfer process: Figure S18
M. Optical characteristics of In2Sez on mica substrate: Figure S19 \& S20 


\section{A. Growth setup and morphology of $\operatorname{In}_{2} \mathrm{Se}_{3}$ :}
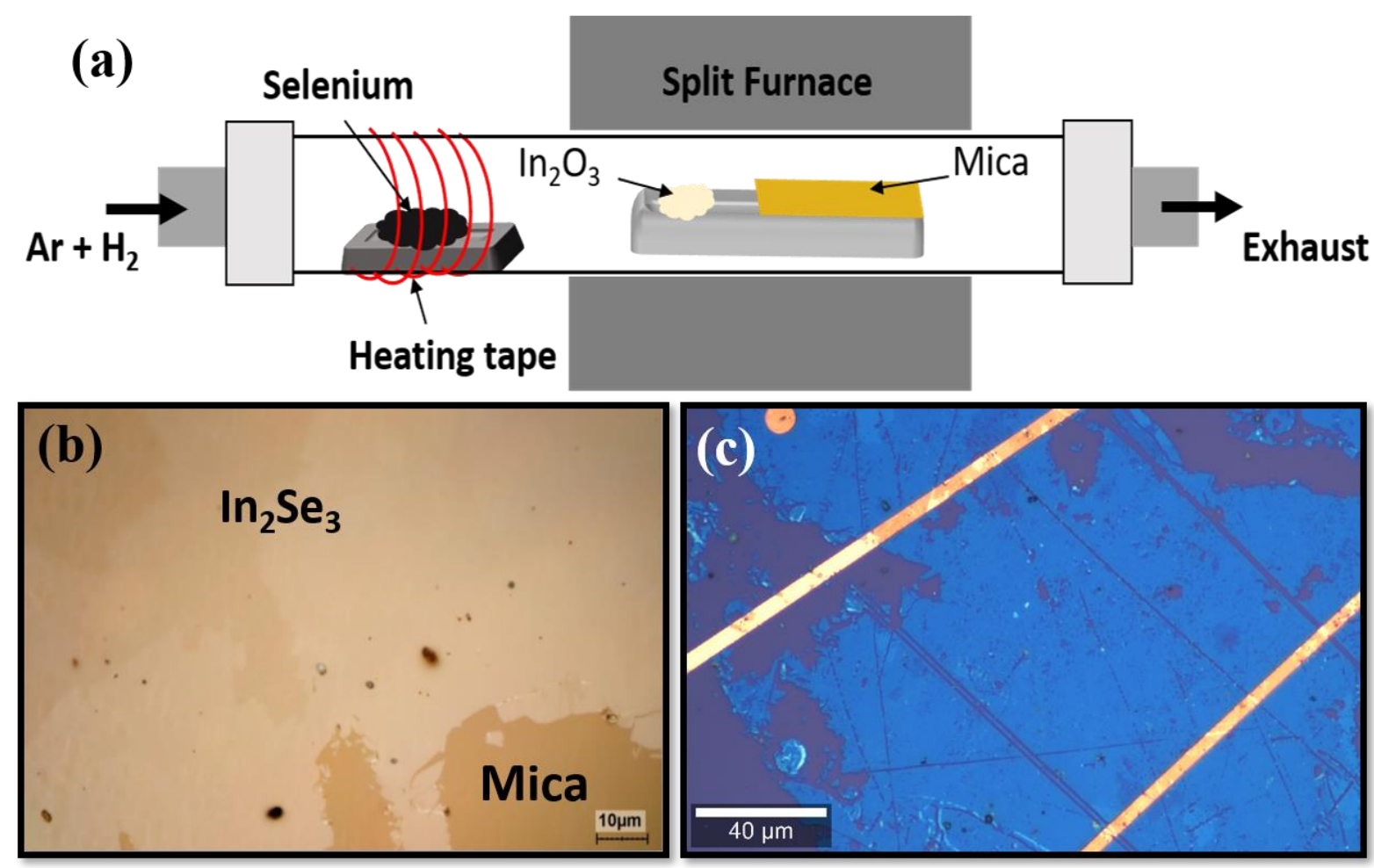

Figure S1: (a) Schematic set-up illustration of the growth process of $\operatorname{In}_{2} \mathrm{Se}_{3}$ using $\operatorname{In}_{2} \mathrm{O}_{3}$ and $\mathrm{Se}$ powder. Optical microscope images of $\mathrm{In}_{2} \mathrm{Se}_{3}$ on (b) mica substrate after growth and (c) $\mathrm{SiO}_{2}$ substrate after wettransfer.
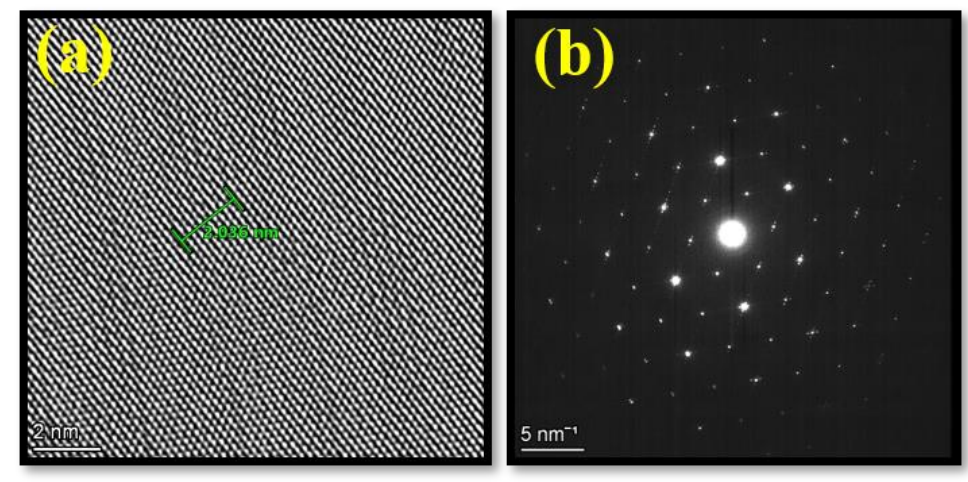

Figure S2: HR-TEM micrograph of (a) as-grown $\operatorname{In}_{2} \mathrm{Se}_{3}$ exhibits good crystal quality with atomic spacings of about $\sim 0.20 \mathrm{~nm}$, attributed to the $\left(\begin{array}{lll}1 & 1 & 0\end{array}\right)$ along the [1120] direction ${ }^{1}$, and (b) SAED of the same also exhibiting high crystaline quality. 


\section{B. Effect of laser dose on $\operatorname{In}_{2} \mathrm{Se}$ :}

To find the effect of time period of laser irradiation on our sample, we have performed a control experiment for different intensities and exposure times, while maintaining the same optical dose, for fair comparisons. The optical dose is defined as:

$$
\text { Optical dose, } D\left(\frac{m J}{\mathrm{~cm}^{2}}\right)=\frac{\text { incident power } * \text { exposure time }}{\text { illuminated area }}\left(\frac{\mathrm{mW} * \mathrm{sec}}{\mathrm{cm}^{2}}\right)
$$

The optical image for various exposure periods and powers is presented in Figure S3(a). Corresponding optical dose is written above each column. Raman maps for the characteristic vibrational modes $\left(\sim 107 \mathrm{~cm}^{-1}\right.$ and $\left.253 \mathrm{~cm}^{-1}\right)$ obtained are depicted in Figure S3(b-c), respectively.

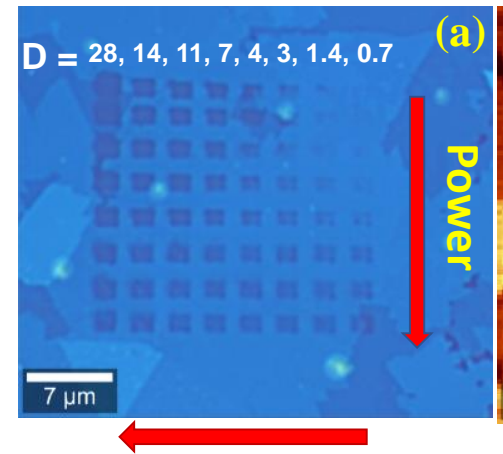

Time

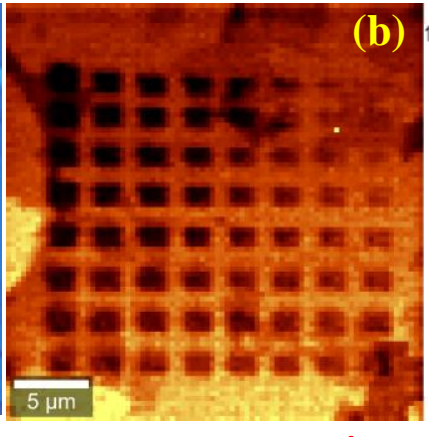

$\omega=107 \mathrm{~cm}^{-1}$

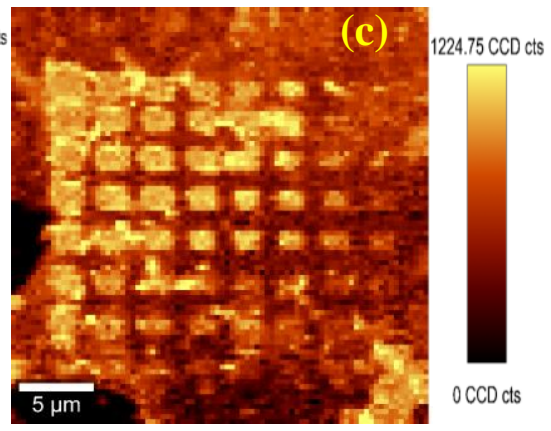

$\omega=253 \mathrm{~cm}^{-1}$

Figure S3: Effect of various laser exposure period and irradiation power on $\operatorname{In}_{2} \mathrm{Se}_{3}$. (a) Optical image; Raman mapping for (b) $107 \mathrm{~cm}^{-1}$ and (c) $253 \mathrm{~cm}^{-1}$ modes.

It is clearly observed that for low power and short time exposures there is almost no effect on $\mathrm{In}_{2} \mathrm{Se}_{3}$, indicates negligible photo-induced local temperature change. However, for high powers one can see a distinct change, even for a very short pulse. Interestingly, for low optical power, and prolonged exposure, we observe materials' conversion and broadening of the exposed area (square boxes). This indicates that the heat dissipation mostly takes place at the cross-plane direction and has strong time-dependent thermal anisotropy for heat conduction, which is typical for $2 \mathrm{D}$ materials. The material's conversion mechanism is schematically illustrated in Figure S4. Previous studies suggest that the local laser heating can weaken certain atomic bonds and destabilize the crystal lattice ${ }^{2}$. Therefore, the heat dissipation in the material is an important factor which can significantly increase the local temperature and can lead to the larger-than-diffraction-limited laser profile of actual exposed region. Moreover, high absorption coefficient of $\operatorname{In}_{2} \mathrm{Se}_{3}\left(\sim 10^{5} \mathrm{~cm}^{-1}\right)$ results in significant absorption of light, and 
high thermal anisotropy leading to strong local heating ${ }^{3}$. The rate of thermal transport away from an optically heated surface depends strongly on the thermal conductivity of the material ${ }^{4}$. It should be noted that the effect of domain-boundary scattering, presence of grain boundaries and phonon impurities in our CVD grown films combinedly lead to the poor heat dissipation, resulting in significant local temperature increment.

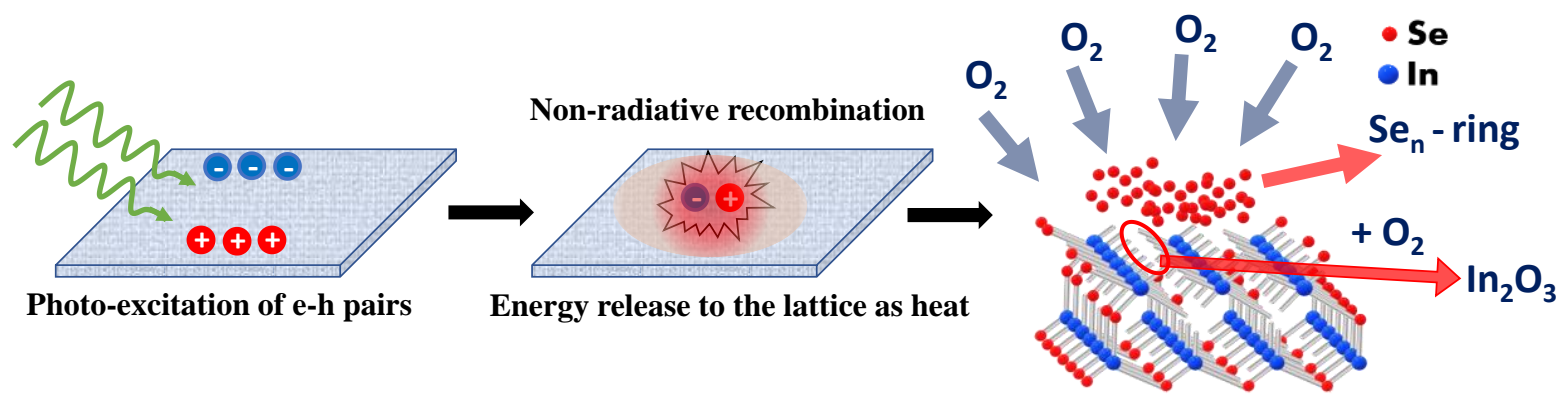

Figure S4: Schematic illustration of the laser-induced thermal annealing (photo-thermal) effect.

\section{Laser induced simulated temperature rise on $\operatorname{In}_{2} S e 3$ :}

To better understand the transient evolution of the surface temperature and heat dissipation in our experiment, we present a finite element method (FEM) based thermal simulation model using COMSOL Multiphysics software package. The model provides the time dependent laserinduced estimated local temperature rise in $\mathrm{In}_{2} \mathrm{Se}_{3}$ on $\mathrm{SiO}_{2} / \mathrm{Si}$ substrate for different values of incident power. The heat diffusion equation was solved by considering a Gaussian-shaped laser beam as the heat source. The thickness of $\mathrm{In}_{2} \mathrm{Se}_{3}$ and $\mathrm{SiO}_{2}$ layer were reserved as $10 \mathrm{~nm}$ and $300 \mathrm{~nm}$ respectively to replicate the actual device structure. The layer-thickness dependent thermal conductivity, absorption coefficient and reflection coefficient were taken from literature ${ }^{5}$. The average simulated temperature (Ts) due to the laser heating was obtained using equation, $\quad \mathrm{T}_{s}=\frac{\int_{0}^{r} \mathrm{~T}^{\prime}(r) Q(r) r d r}{\int_{0}^{r} Q(r) r d r}$

where $\mathrm{T}^{\prime}(r)$ is the local temperature rise for laser heating as a function of distance $r$ from the center of laser spot. $\mathrm{Q}(\mathrm{r})$ represents the volumetric Gaussian laser heat source, and can be described as,

$$
Q(r)=\frac{P_{a b}}{\pi r_{0}^{2}} e^{-\left(\frac{r^{2}}{r_{0}^{2}}\right)} \cdot e^{-\alpha|d|}
$$


where $\mathrm{P}_{\mathrm{ab}}$ is the absorbed laser power, $\mathrm{d}$ is the layer thickness, and $\mathrm{r}_{0}$ is the beam width. The absorbed laser power can be written as, $\mathrm{P}_{a b}=(1-\mathrm{R}) \alpha \mathrm{P}_{0}$, where $\mathrm{P}_{0}$ is the incident laser power, $\mathrm{R}$ and $\alpha$ are the $\operatorname{In}_{2} \mathrm{Se}_{3}$ reflection and absorption coefficients, respectively.

The time-dependent heat transfer was solved using the following equation:

$$
\rho C_{s p}\left(\frac{\partial T}{\delta t}+V_{t r} . \nabla T\right)+\nabla \cdot\left(q_{c}+q_{r}\right)=Q_{i n}
$$

where, $\rho, C_{\text {sp }}$ and $\alpha_{T h}$ are the density, specific heat capacity and thermal expansion coefficient of the materials, respectively; $\mathrm{T}$ is the temperature; $\mathrm{q}_{\mathrm{c}}$ and $\mathrm{q}_{\mathrm{r}}$ are the heat flux by conduction and radiation; $Q_{\text {in }}$ is the heat source for laser illumination.

A simulated surface temperature distribution is shown in Figure S5(a) with a planner projection (Figure S5(b)). The time-dependent local temperature rise for two laser powers are exhibited in Figure S5(c-d). For the high laser intensity, the temperature rapidly rises and can initiate the material's conversion process, but for low intensity, one required much longer timescale to reach that temperature. The time-dependent nonlinear heating predicted by the model for longer time scale is mostly dominated by in-plane heat flow and saturates as the system reaches thermal equilibrium. It is reported that at high temperatures the specific heat is expected to saturate, which might be the reason for saturation of the local temperature rise with time, showing good agreement with the experimental data. In a nutshell, a smaller laser power for longer exposure time allows better heat dissipation in the lateral directions, identified as overdose for the desired structure (square boxes in Figure S3). However, low-power and low exposure time indicates negligible optical heating, suggesting under dose for the patterning. Therefore, moderate power $(>5-6 \mathrm{~mW})$ and low-exposure time is optimum to engineer in-plane heterostructure devices. 

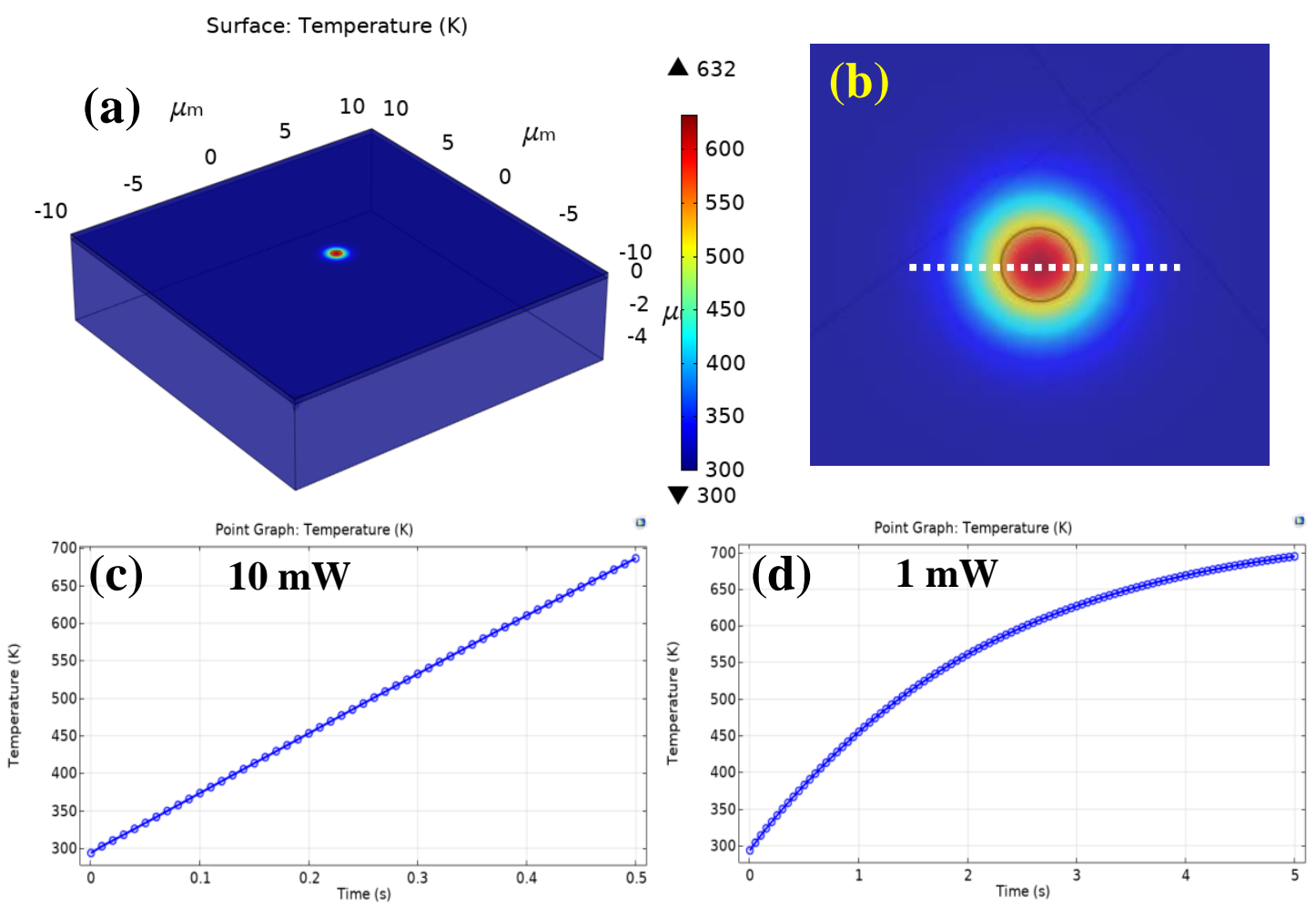

Figure S5: (a) Surface temperature profile on $10 \mathrm{~nm} \mathrm{In}{ }_{2} \mathrm{Se}_{3}$ layer mounted in $\mathrm{SiO}_{2} / \mathrm{Si}$ substrate obtained from the 3D simulation model. (b) The planar projection of the surface temperature on $\operatorname{In}_{2} \mathrm{Se}_{3}$ obtained from figure (a). The temperature was estimated from the maxima of the gaussian beam profile, taken along the white dotted line. Time-dependent simulated temperature evolution for (c) $10 \mathrm{~mW}$, and (d) 1 $\mathrm{mW}$ laser power.

\section{Effect of ex-situ anneling:}

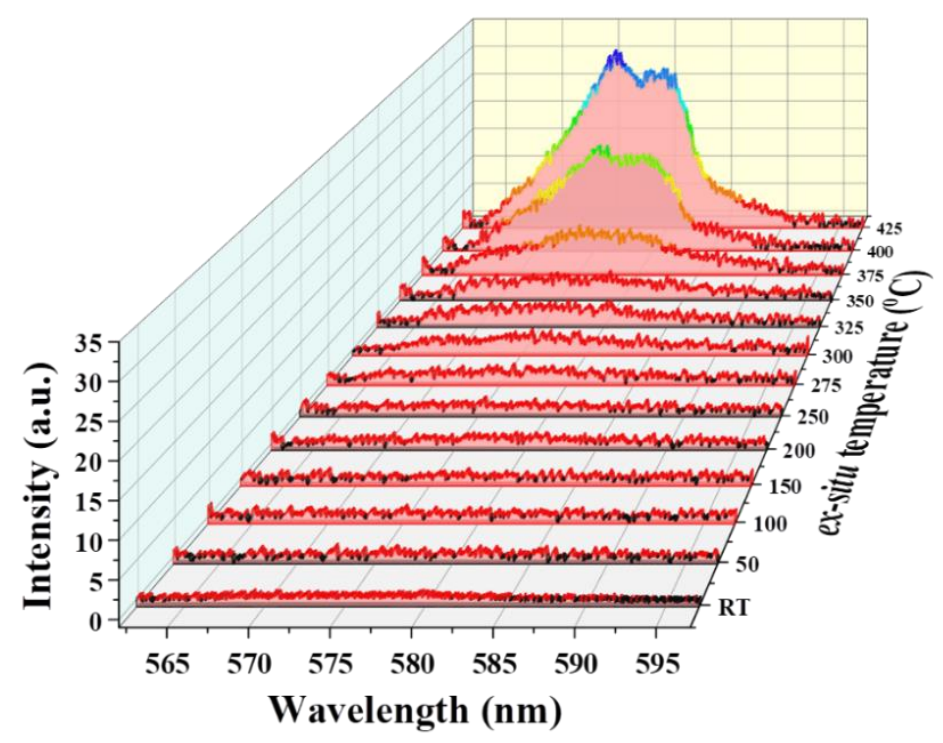

Figure S6: Photoluminescence spectra of $\mathrm{In}_{2} \mathrm{Se}_{3}$ for different annealing temperatures under airatmosphere. Each PL spectrum was measured following a $30 \mathrm{~min}$ thermal annealing in ambience. It is observed that above $300{ }^{\circ} \mathrm{C}$, the PL emission for $\operatorname{In}_{2} \mathrm{O}_{3}$ started to become apparent. This indicates that the material transformation is dictated by thermal excitation in agreement with the modelling [Figure S5] and control experiments [Figure S3]. 


\section{E. Effect of gaussian laser beam prolife on materials conversion:}

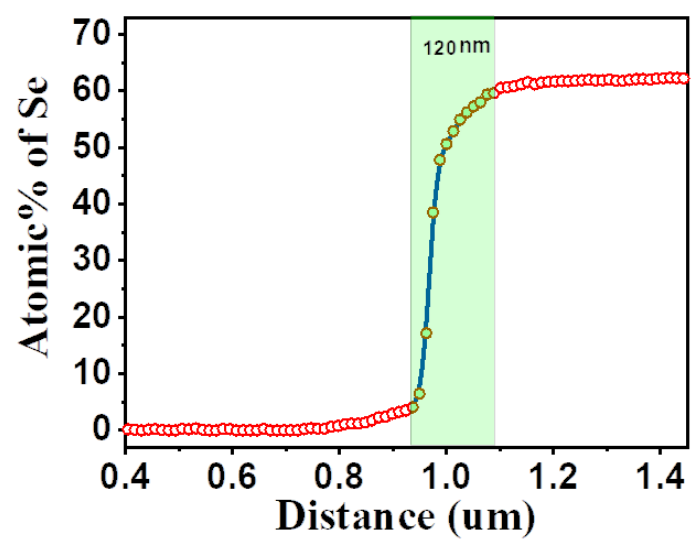

Figure S7: The line profile for Se atomic wt $\%$ across the $\operatorname{In}_{2} \mathrm{O}_{3}-\mathrm{In}_{2} \mathrm{Se}_{3}$ interface was estimated from the STEM-EDS analysis.

\section{F. Raman maps for low intensity laser illumination:}
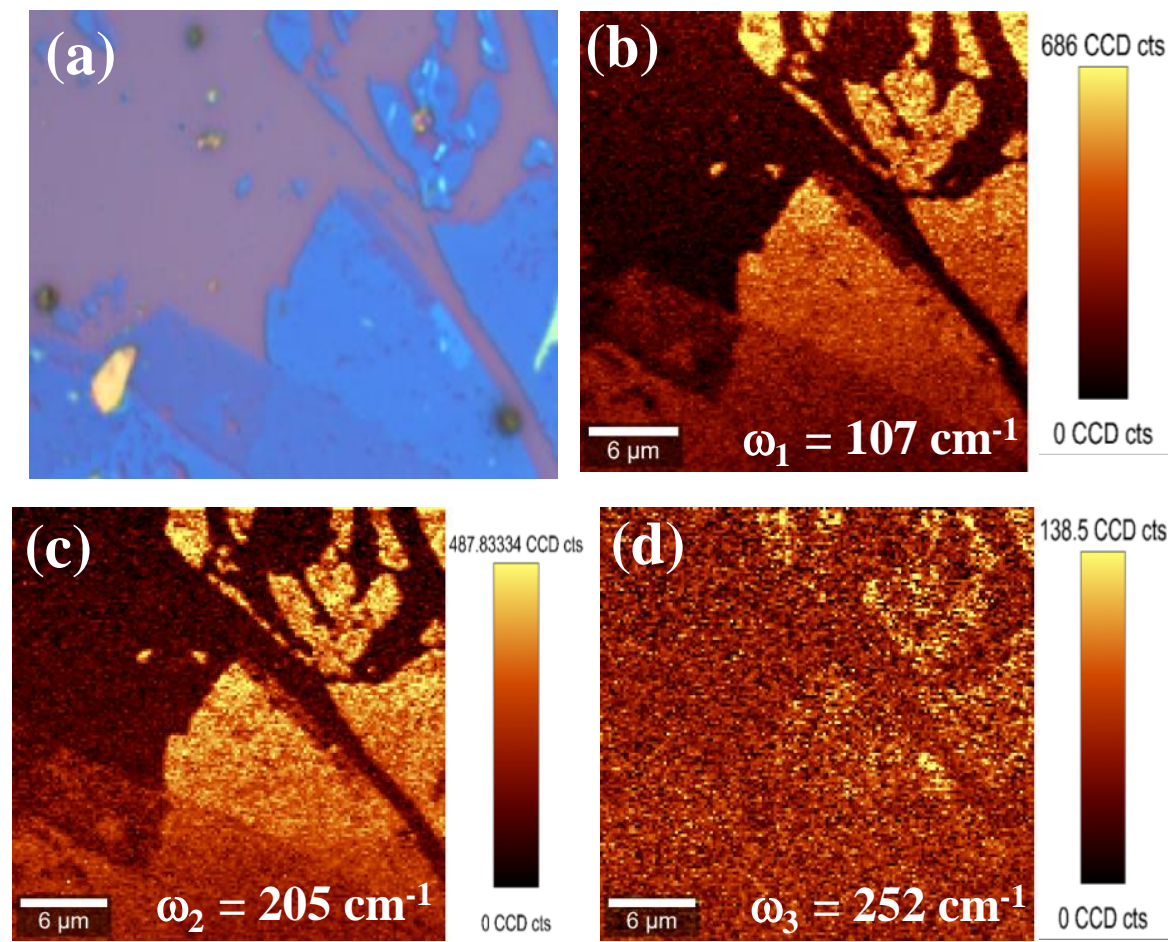

138.5 CCD cts

Figure S8: (a) Optical image of $\mathrm{In}_{2} \mathrm{Se}_{3}$ film on $\mathrm{SiO}_{2}$ surface. Raman mapping of the selected region for peak (b) 107, (c) 205 and (d) $252 \mathrm{~cm}^{-1}$, indicating that without strong (above the threshold power) laser illumination, the $\mathrm{In}_{2} \mathrm{Se}_{3}$ films retained their characteristics properties. There is no evidence of -Ses- rings formation without reasonable laser illumination (Figure d). 


\section{G. Laser exposure intensity calibration by KPFM:}

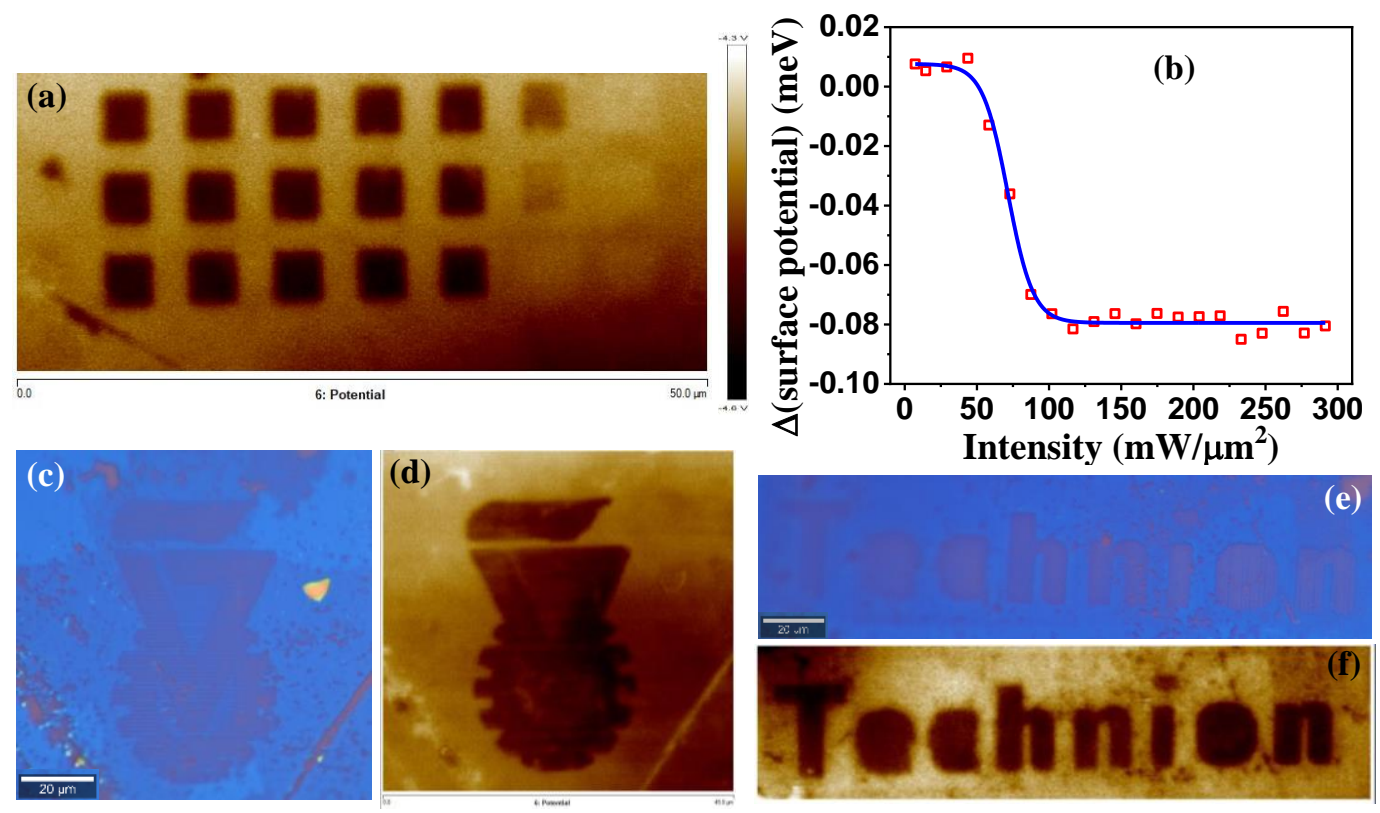

Figure S9: (a) Kelvin-probe force microscopy (KPFM) image acquired on the same area, where PL and Raman mapping was performed. (b) Variation of the potential on the exposed area compared to the background $\mathrm{In}_{2} \mathrm{Se}_{3}$ film is plotted as the function of irradiated laser intensity. (c) Optical and (d) KPFM image of Technion-logo, and (e) optical and (f) KPFM image of Technion (letters) written using optothermal effect on $\operatorname{In}_{2} \mathrm{Se}_{3}$, establishing the flexibility of the method to design arbitrary shaped circuitry and offer the prospect for several technological applications, such as on-demand electronic circuitries, patterns/design parallel stitching etc.

\section{H. Comparison of laser exposure effect on sample and substrate:}
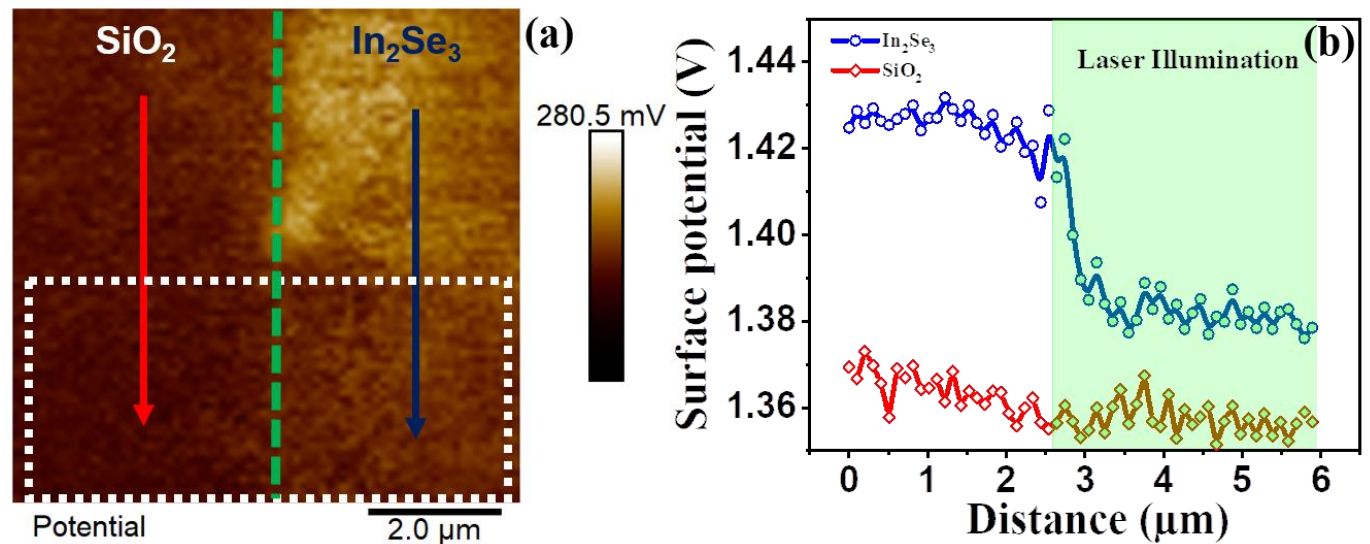

Figure S10: (a) surface potential map of the $\mathrm{In}_{2} \mathrm{Se}_{3}$ on $\mathrm{SiO}_{2}$ surface after selective exposure. The white dotted box region was exposed. The green dashed line separates the $\mathrm{In}_{2} \mathrm{Se}_{3}$ from the $\mathrm{SiO}_{2}$ background. The left side of green dashed lines represents the base $\mathrm{SiO}_{2}$, whereas the right side represents $\mathrm{In}_{2} \mathrm{Se}_{3}$ layers. (b) The potential profiles are obtained along the arrow line (line colours kept same as corresponding profile colour for easy understanding). 


\section{Surface potential, electric field and conductivity of FETs:}

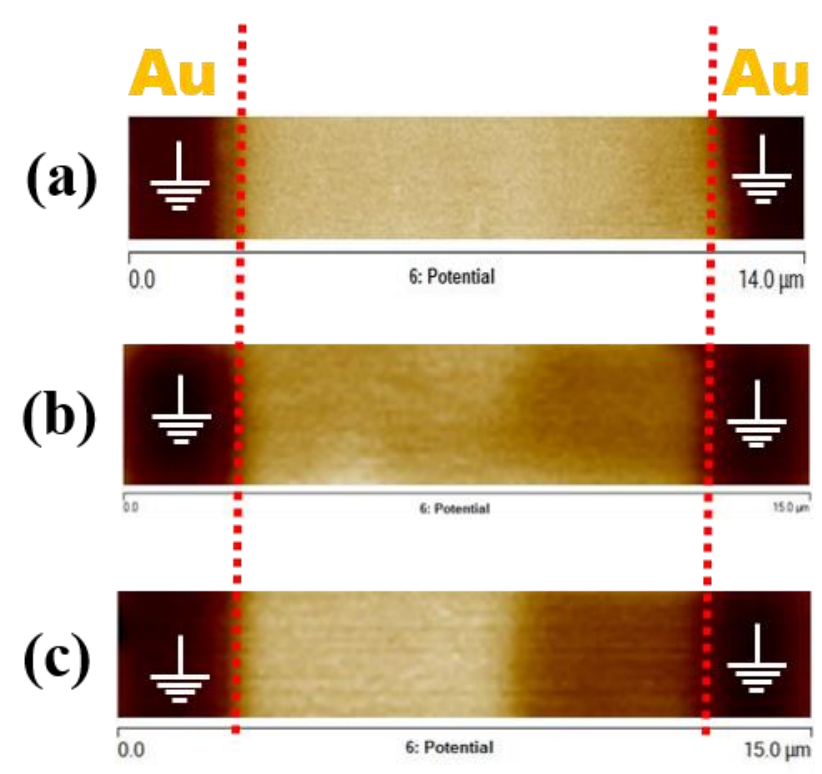

Figure S11: Surface potential mapping of the fabricated FETs, consisting the channel materials as, the (a) pristine $\mathrm{In}_{2} \mathrm{Se}_{3}$, (b) converted $\mathrm{In}_{2} \mathrm{O}_{3}$ and (c) coplanar heterojunction of $\operatorname{In}_{2} \mathrm{Se}_{3}-\mathrm{In}_{2} \mathrm{O}_{3}$, under equilibrium condition. The perpendicular red-coloured dotted lines are indicating the metalsemiconductor interfaces. The brownish area presents the converted $\operatorname{In}_{2} \mathrm{O}_{3}$, while the whitish area retained as $\operatorname{In}_{2} \mathrm{Se}_{3}$.
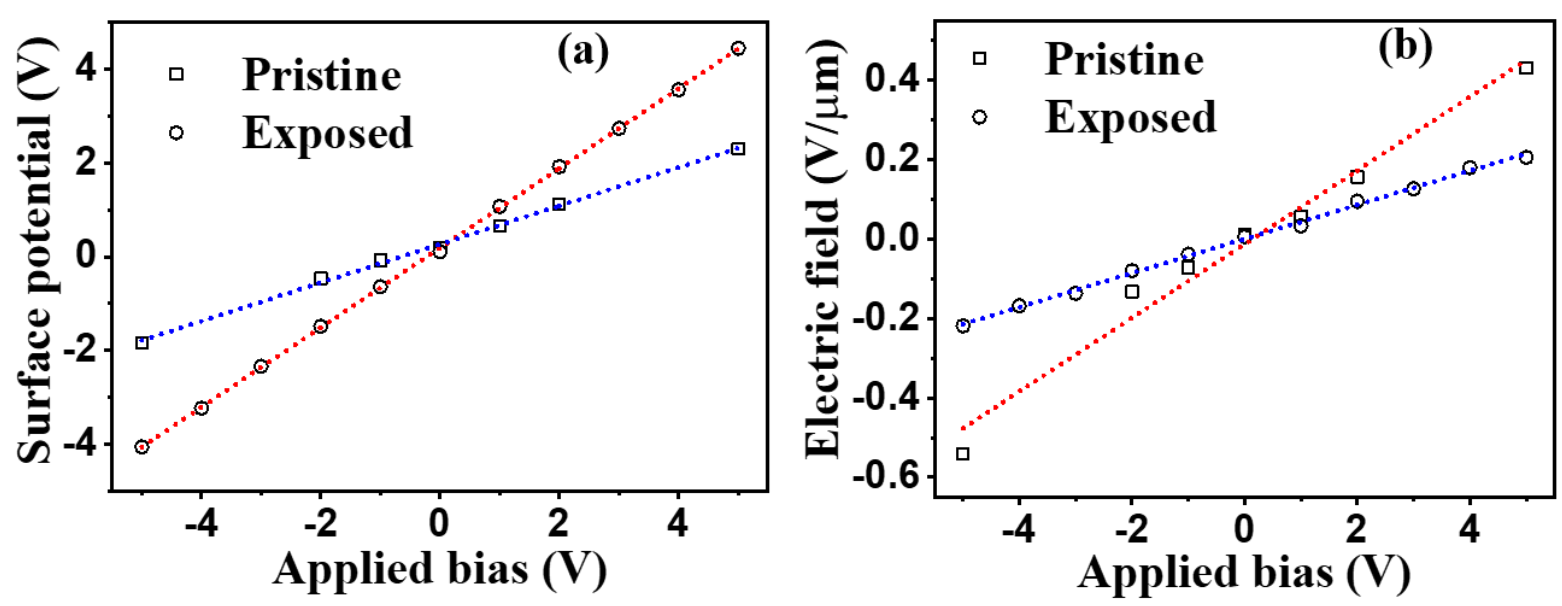

Figure S12: Variation of (a) localized surface potential (measured at centre of FET channel) and (b) estimated electric field (in FET channel), as a function of applied bias. The measured surface potential and estimated electric field are progressively increasing with applied bias for both cases, but are significantly smaller for illuminated FET pointing to higher carrier flow due to higher conductivity of $\mathrm{In}_{2} \mathrm{O}_{3}$, as well as Schottky barrier modification. 


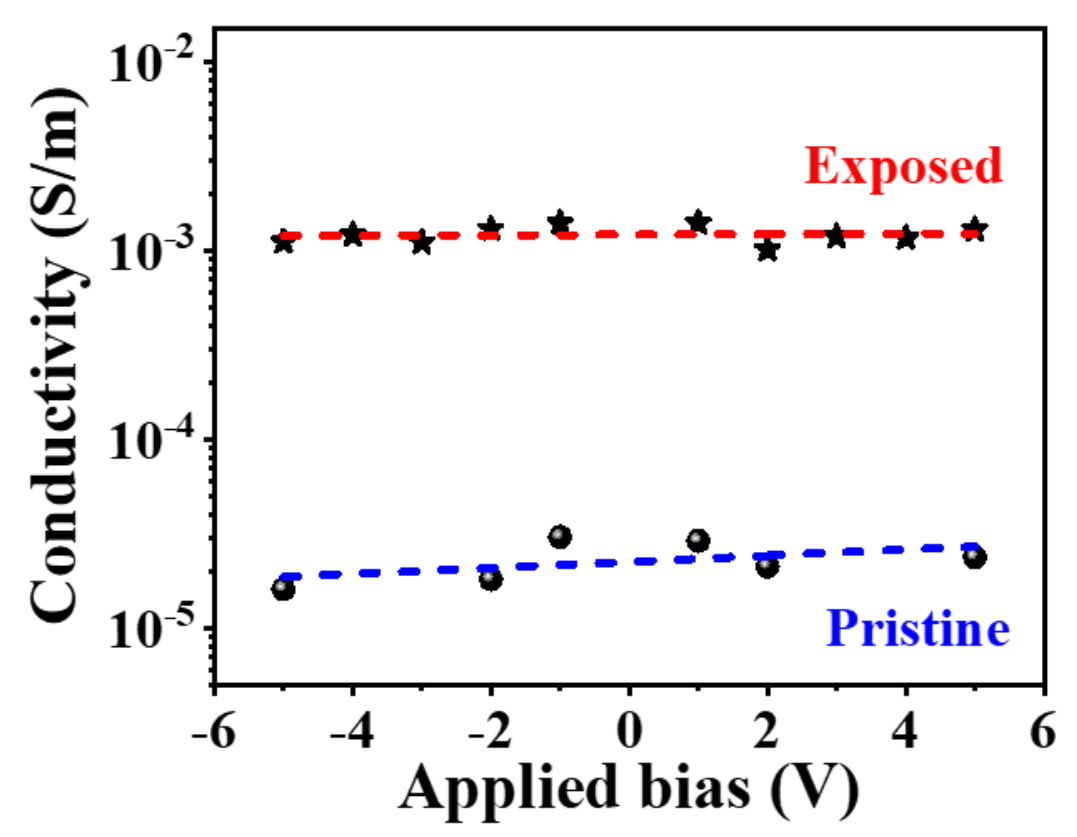

Figure S13: Deduced conductivity of the pristine channel and the laser irradiated channel using KPFM, as a function of applied bias, shows nearly two-order enhancement.

\section{J. Photoresponse characteristics of the FETs:}
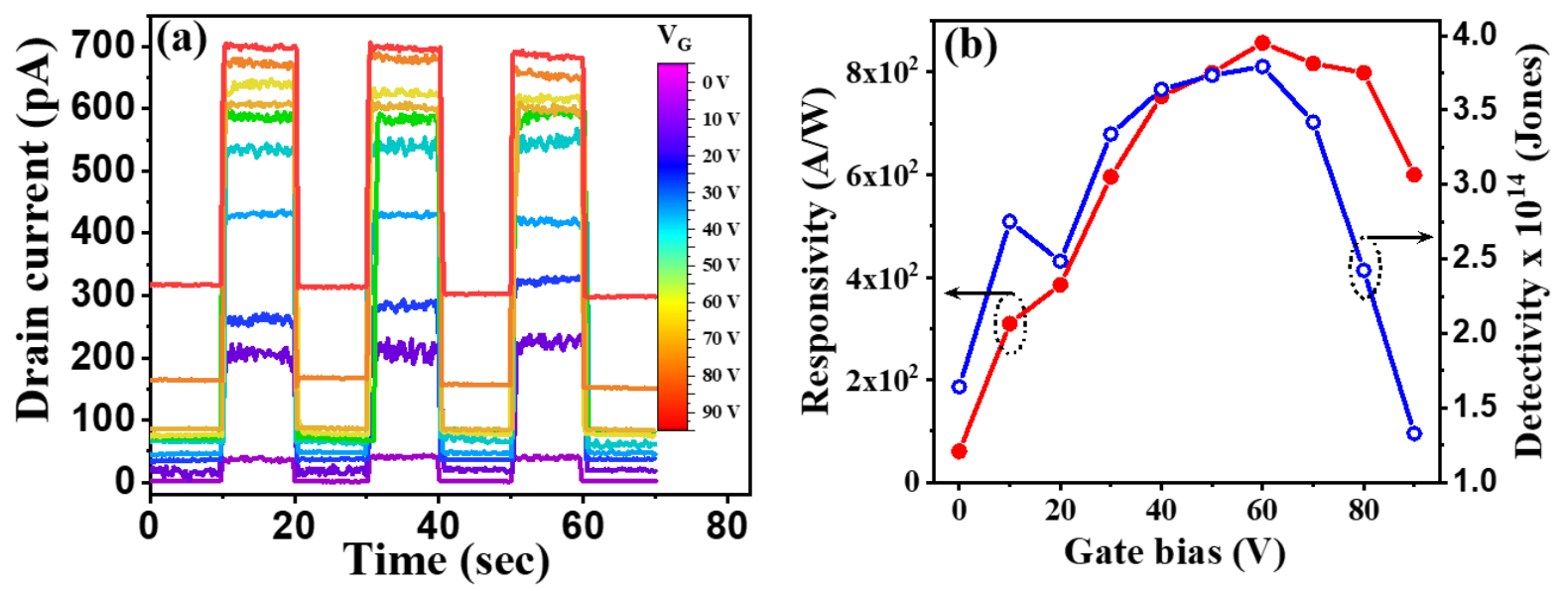

Figure S14: (a) Time-dependent photo-response under periodic illumination for diffrent gate bias. (b) Calculated Responsivity and detectivity of the fabricated heterojunction plotted against the applied gate bias.

The responsivity of a photodetector device can be calculated using the following equation ${ }^{6}$,

$$
\Re(\lambda)=\frac{J_{\text {Photo }}(\lambda)}{P_{d}}
$$

Where, $J_{\text {Photo }}$ is the photocurrent density and $P_{d}$ is the incident power density. 
Detectivity $\left(D^{*}\right)$, which is another important parameter of a photodetector, represents the ability to detect weak optical signals from noisy background and can be expressed as ${ }^{7}$,

$$
D^{*}=\frac{\mathfrak{R}(\lambda)}{\sqrt{q . J_{d a r k}}}
$$

Where, $J_{\text {dark }}$ is the dark current density and $q$ is the electronic charge.
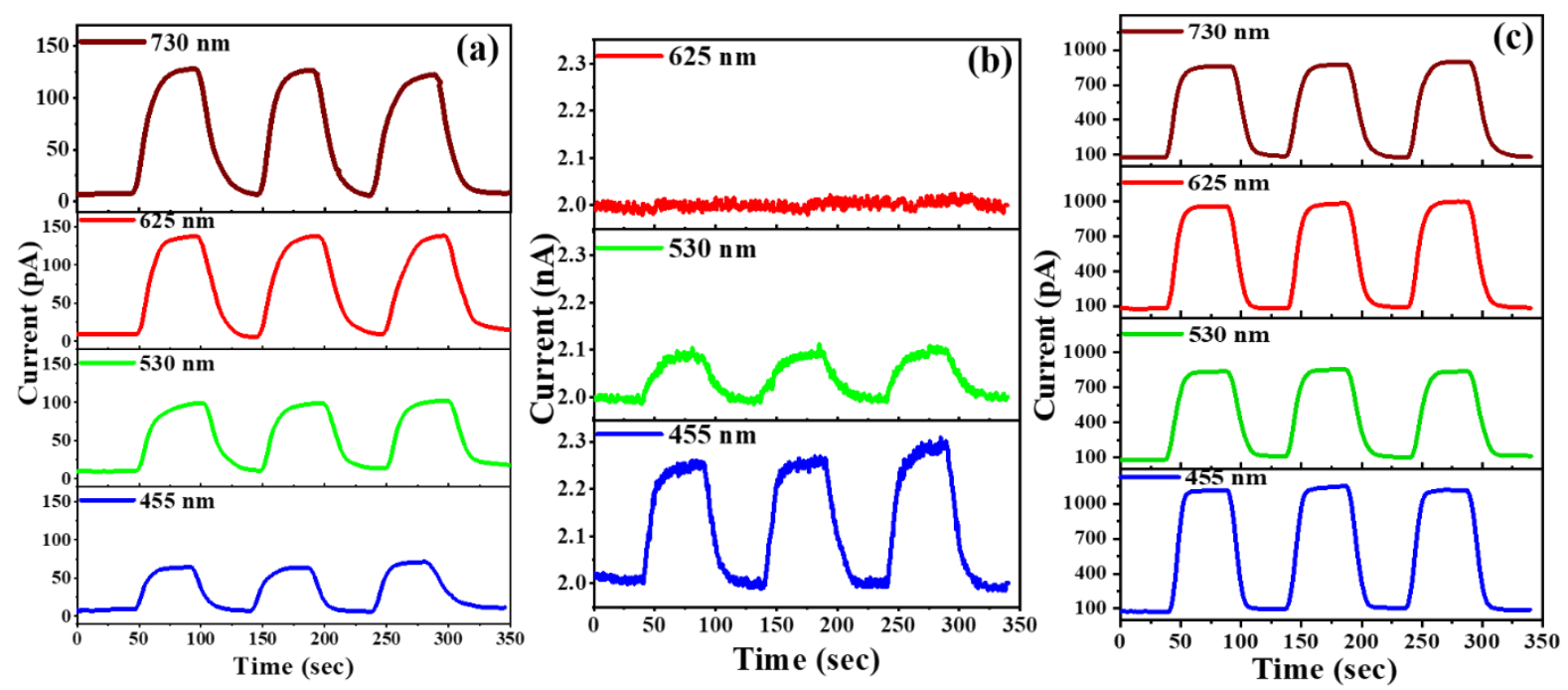

Figure S15: The photo-switching behaviours of the (a) $\mathrm{In}_{2} \mathrm{Se}_{3}$, (b) $\mathrm{In}_{2} \mathrm{O}_{3}$ and (c) $\mathrm{In}_{2} \mathrm{Se}_{3}-\mathrm{In}_{2} \mathrm{O}_{3}$ heterojunction devices, under periodic illumination. Each graph is color-coded as the incident light color. The limits for $\mathrm{Y}$ axes scale are kept same for all wavelengths for easy comparison.

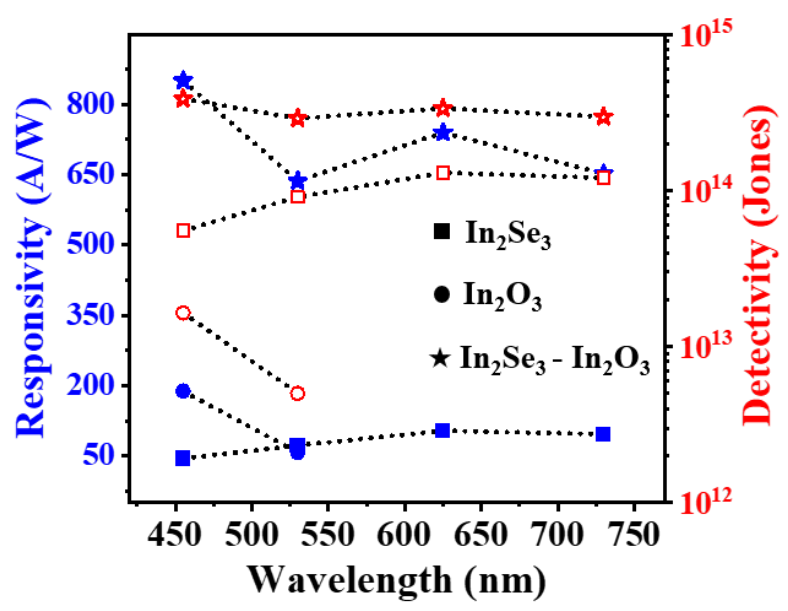

Figure S16: Wave-length dependent photo-responsivity (solid symbols) and photo-detectivity (open symbols) data for all three devices. The dotted lines are drawn for eye-guiding. 


\section{K. 2D layer transfer illustration:}

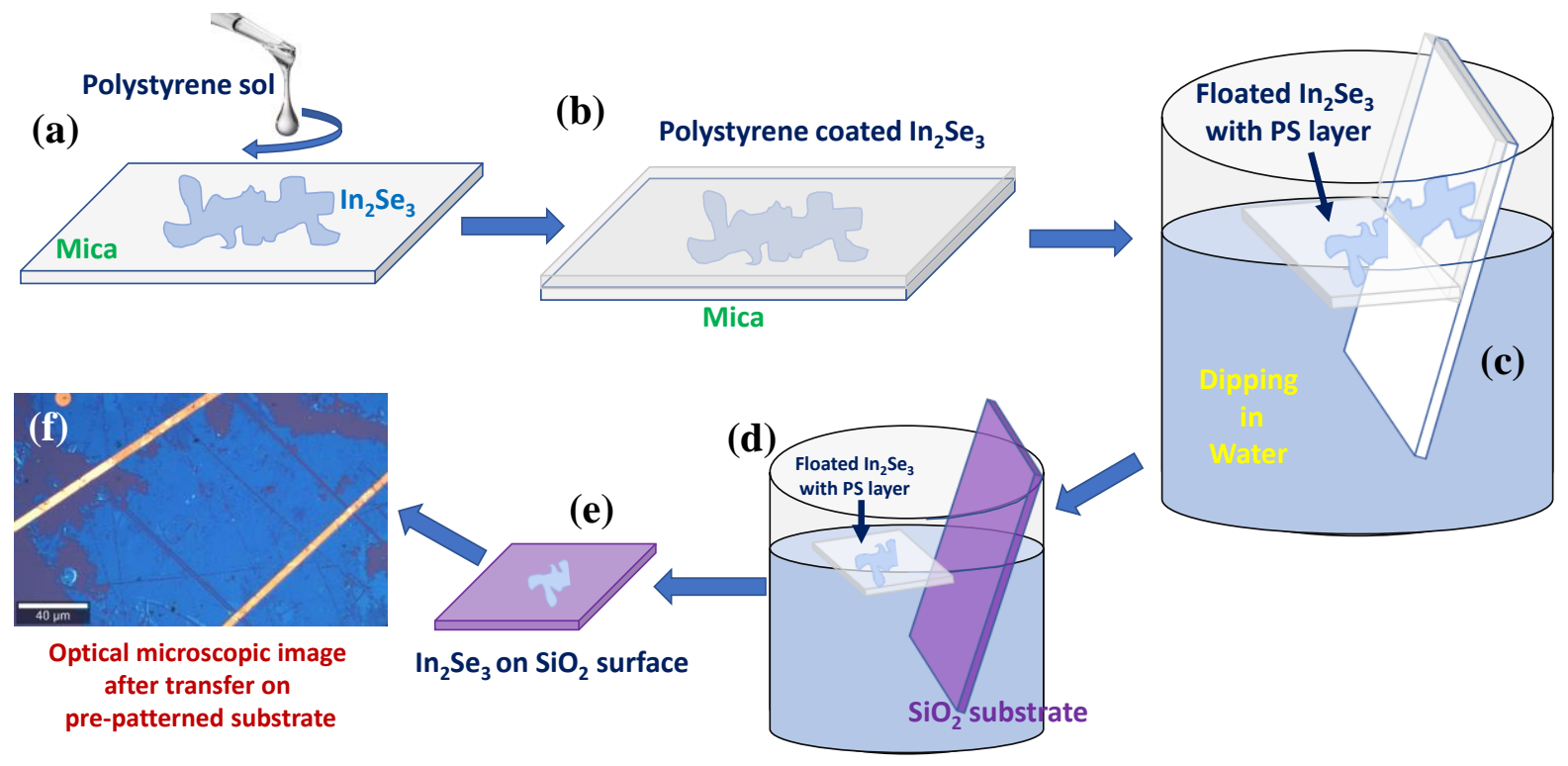

Figure S17: Schematic flow-chart of the wet-transfer method of $\operatorname{In}_{2} \mathrm{Se}_{3}$ from growth substrate to the targeted one.

\section{Raman characteristics of $\operatorname{In}_{2} \mathrm{Se}_{3}$ before and after wet-transfer process:}

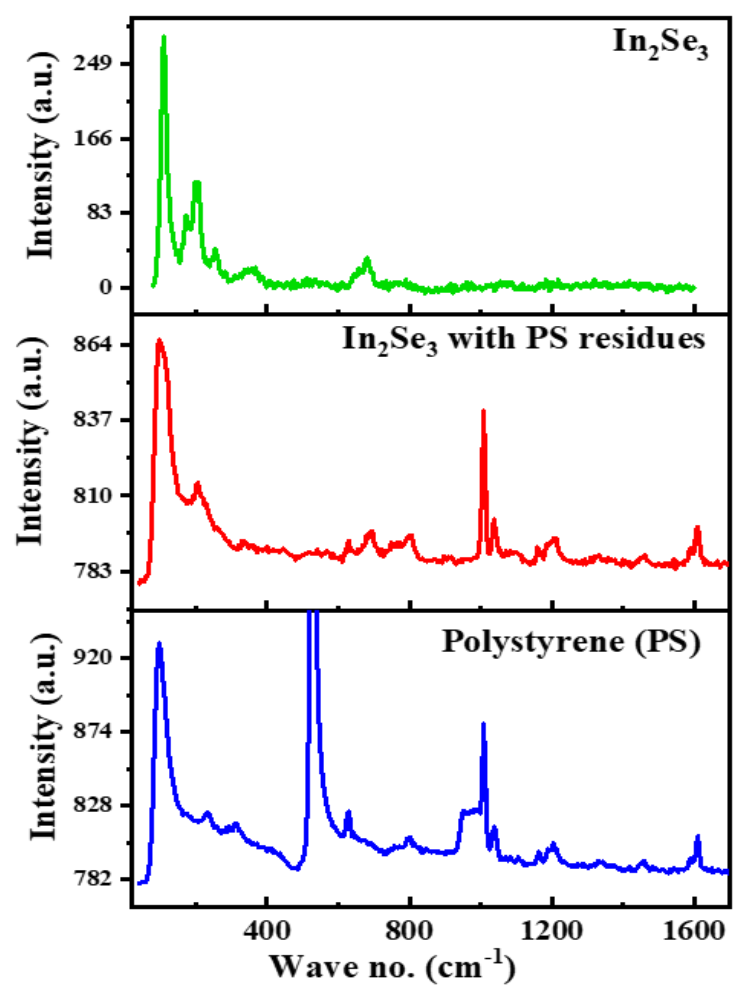

Figure S18: Comparative Raman spectra for polystyrene (PS), $\mathrm{In}_{2} \mathrm{Se}_{3}$ with PS residues and $\operatorname{In}_{2} \mathrm{Se}_{3}$ after PS removal, on $\mathrm{SiO}_{2}$ substrate. 


\section{Optical characteristics of $\operatorname{In}_{2} \mathrm{Se}_{3}$ on mica substrate:}

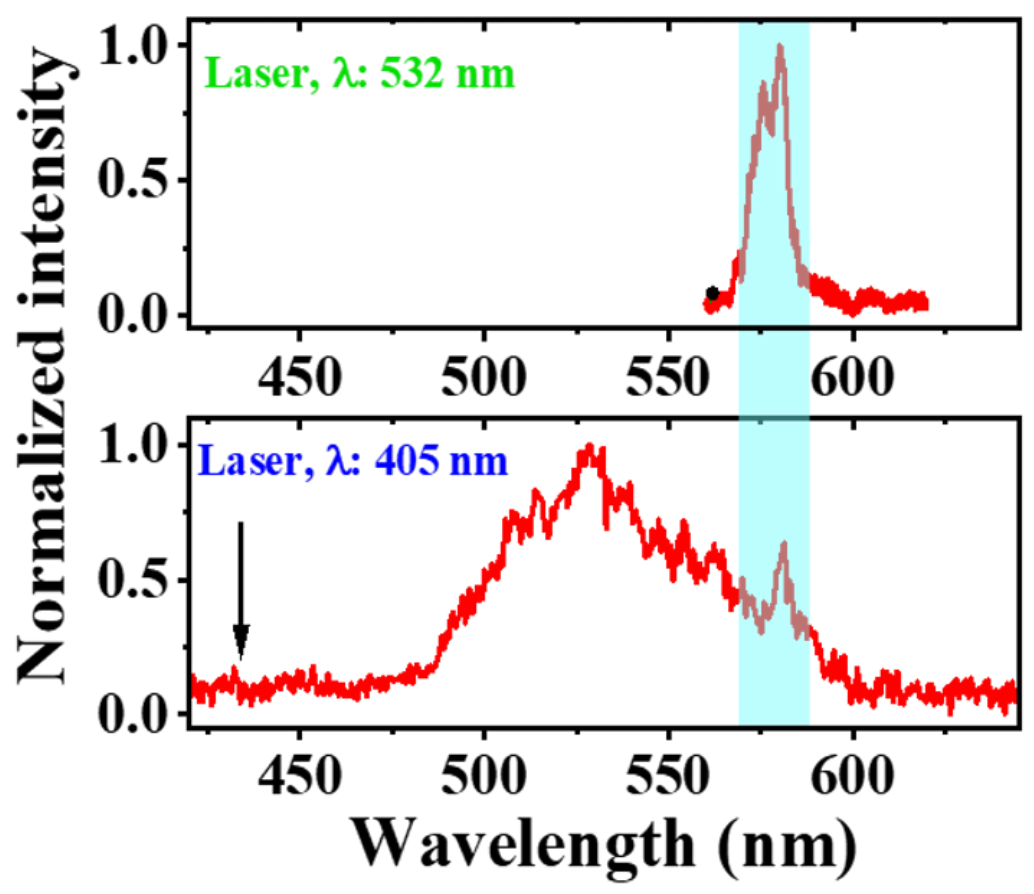

Figure S19: PL spectra of CVD-grown $\operatorname{In}_{2} \mathrm{Se}_{3}$ on growth (mica) substrate using the excitation wavelength of $532 \mathrm{~nm}$ (top) and $405 \mathrm{~nm}$ (bottom). The arrow indicates the expected position of graphitic Raman signals using $405 \mathrm{~nm}$ excitation.
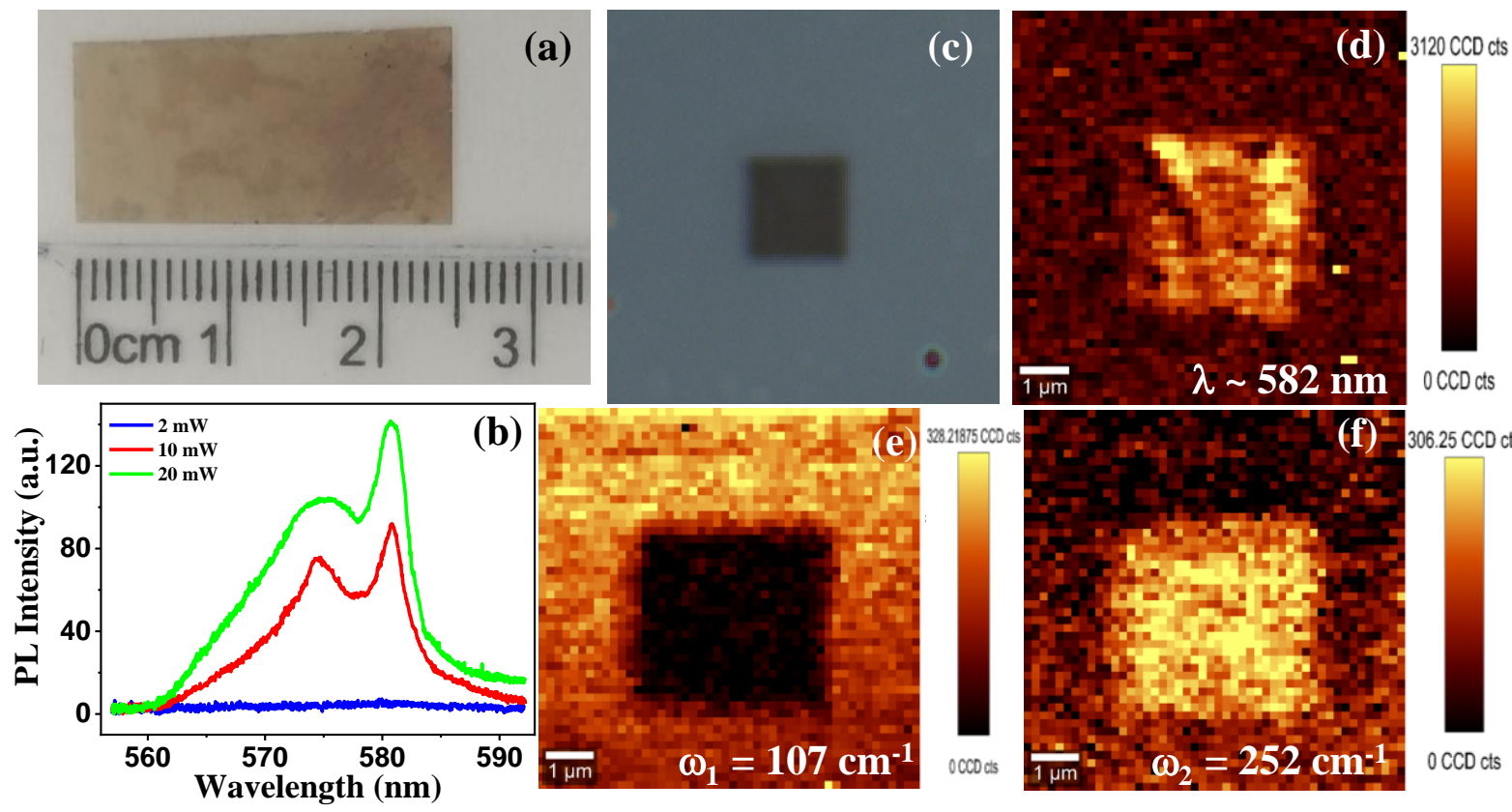

Figure S20: (a) Optical image of $\operatorname{In}_{2} \mathrm{Se}_{3}$ on growth substrate (mica). (b) PL spectra, acquired for different laser powers. (c) Optical image of the laser written box ( $4 \times 4 \mu \mathrm{m}$ ) on $\mathrm{In}_{2} \mathrm{Se}_{3}$ film on growth substrate. (d) PL (for $582 \mathrm{~nm}$ ) and (e-f) Raman (for 107 and $252 \mathrm{~cm}^{-1}$ ) maps on the same box were acquired. 
References:

(1) Jacobs-Gedrim, R. B.; Shanmugam, M.; Jain, N.; Durcan, C. A.; Murphy, M. T.; Murray, T. M.; Matyi, R. J.; Moore, R. L.; Yu, B. Extraordinary Photoresponse in Two-Dimensional $\operatorname{In}_{2} \mathrm{Se}_{3}$ Nanosheets. ACS Nano 2014, 8 (1), 514-521.

(2) Igo, J.; Gabel, M.; Yu, Z. G.; Yang, L.; Gu, Y. Photodefined In-Plane Heterostructures in TwoDimensional $\mathrm{In}_{2} \mathrm{Se}_{3}$ Nanolayers for Ultrathin Photodiodes. ACS Appl. Nano Mater. 2019, 2 (10), 6774-6782.

(3) Yalon, E.; Aslan, Ö. B.; Smithe, K. K. H.; McClellan, C. J.; Suryavanshi, S. V.; Xiong, F.; Sood, A.; Neumann, C. M.; Xu, X.; Goodson, K. E.; Heinz, T. F.; Pop, E. Temperature-Dependent Thermal Boundary Conductance of Monolayer $\mathrm{MoS}_{2}$ by Raman Thermometry. ACS Appl. Mater. Interfaces 2017, 9 (49), 43013-43020.

(4) Kargar, F.; Coleman, E. A.; Ghosh, S.; Lee, J.; Gomez, M. J.; Liu, Y.; Magana, A. S.; Barani, Z.; Mohammadzadeh, A.; Debnath, B.; Wilson, R. B.; Lake, R. K.; Balandin, A. A. Phonon and Thermal Properties of Quasi-Two-Dimensional $\mathrm{FePS}_{3}$ and $\mathrm{MnPS}_{3}$ Antiferromagnetic Semiconductors. ACS Nano 2020, 14 (2), 2424-2435.

(5) Zhou, S.; Tao, X.; Gu, Y. Thickness-Dependent Thermal Conductivity of Suspended TwoDimensional Single-Crystal $\mathrm{In}_{2} \mathrm{Se}_{3}$ Layers Grown by Chemical Vapor Deposition. J. Phys. Chem. C 2016, 120 (9), 4753-4758.

(6) Mukherjee, S.; Maiti, R.; Katiyar, A. K.; Das, S.; Ray, S. K. Novel Colloidal MoS 2 Quantum Dot Heterojunctions on Silicon Platforms for Multifunctional Optoelectronic Devices. Sci. Rep. 2016, $6(1), 29016$.

(7) Mukherjee, S.; Das, K.; Das, S.; Ray, S. K. Highly Responsive, Polarization Sensitive, SelfBiased Single $\mathrm{GeO}_{2}-\mathrm{Ge}$ Nanowire Device for Broadband and Low Power Photodetectors. ACS Photonics 2018, 5 (10), 4170-4178. 